\title{
ALS antisense oligonucleotides
}

\section{By Lauren Martz, Staff Writer}

Isis Pharmaceuticals Inc. has teamed up with a trio of academic groups to develop antisense therapeutics for the largest subset of patients with amyotrophic lateral sclerosis, ${ }^{1-3}$ and all three teams have converged on a common mechanism of neurotoxicity caused by carrying hexanucleotide repeat expansions in the C9orf72 gene. Antisense oligonucleotides targeting the repeats decreased the toxicity in vitro. The teams now need to determine whether the reduced toxicity correlates with decreased neurodegeneration in patients.

Amyotrophic lateral sclerosis (ALS) is a neurological disorder that involves muscle wasting, stiffness and spasticity due to loss of motor

"Each lab is doing unique experiments, and we were fortunate that the results from the different labs triangulated. We have consistently seen that the antisense oligonucleotides reverse RNA foci formation and reverse the transcriptional problems seen in ALS."

-C. Frank Bennett, Isis Pharmaceuticals Inc. neurons. ALS often overlaps with frontotemporal lobar dementia (FTLD), a disease that involves neuronal degeneration in the frontal and temporal cortices.

Both diseases can occur as inherited familial disorders or sporadically with no known familial link.

The GGGGCC repeat expansion in the first intron of C9orf72 (chromosome 9 open reading frame 72) is the most common genetic cause of ALS. ${ }^{4,5}$ It has been identified in more than $40 \%$ of familial ALS and FTLD cases and in at least 8\% of sporadic cases.

Nevertheless, the mechanistic link between the expansion and neuronal toxicity remained a mystery.

To identify the root causes of neurotoxicity in C9orf72 ALS and FTLD, Isis teamed up with three academic teams.

"Each lab is doing unique experiments, and we were fortunate that the results from the different labs triangulated. We have consistently seen that the antisense oligonucleotides reverse RNA foci formation and reverse the transcriptional problems seen in ALS," said C. Frank Bennett, SVP of research at Isis.

RNA foci are long RNA repeat expansions that have folded into stable structures. The foci sequester RNA-binding proteins required for normal cellular transcription and splicing. The result is disruption in normal gene expression and protein function.
In a paper published in Science Translational Medicine, Robert Baloh and colleagues reported that RNA foci form in cells from patients with C9orf 72 ALS and that blocking the hexanucleotide repeats with antisense oligos decreased foci formation.

Baloh is director of neuromuscular medicine in the Department of Neurology and director of the ALS program at the Cedars-Sinai Medical Center. His team also included researchers from the University of California, Los Angeles, the Mayo Clinic, the University of California, San Diego, the Washington University in St. Louis School of Medicine and Isis.

The team studied motor neurons derived from ALS patient induced pluripotent stem (iPS) cells carrying the C9orf72 hexanucleotide repeat expansions and found signs of RNA toxicity including RNA foci formation in the neurons.

The foci often colocalized with RNA-binding proteins including heterogeneous nuclear ribonucleoprotein A1 (HNRNPA1) and purine-rich element binding protein A (PURA). This colocalization altered expression of genes involved in cellular processes including cell adhesion, synaptic transmission and neuronal differentiation.

The patient-derived cells also had decreased electrical excitability when depolarized compared with cells from healthy controls.

The Cedars-Sinai group treated the patient-derived neurons with antisense oligos targeting either the C9orf 72 gene in general or the repeat region specifically. Both approaches prevented foci formation and partially corrected gene expression, and they increased electrical excitability compared with scrambled antisense oligos.

In a separate study published in Neuron, Jeffrey Rothstein, Rita Sattler and colleagues studied the effects of the repeat expansion using neurons derived from iPS cells from patients with C9orf72 ALS as well as postmortem patient brain tissue.

Rothstein is director for the Brain Science Institute, a professor of neurology and neuroscience and director of the Robert Packard Center for ALS Research at The Johns Hopkins University School of Medicine. Sattler is an assistant professor in the Department of Neurology and a principal scientist in the NeuroTranslational Drug Discovery Program at the Johns Hopkins University School of Medicine.

The paper also included researchers from the University of Helsinki, the Mayo Clinic, the National Institute on Aging and Isis.

The Johns Hopkins team also found the presence of nuclear RNA foci. The foci colocalized with a different RNA-binding protein-adenosine deaminase RNA-specific B2 (ADARB2) — and the binding and sequestering of ADARB2 altered gene expression profiles in both the patient-derived iPS cells and the postmortem tissues.

The group also found that glutamate-induced excitotoxicity, a feature of ALS, was enhanced in the cells from patients but not in cells from healthy controls. In iPS cell-derived neurons from healthy controls, knockout of ADARB2 also elevated glutamate-induced excitotoxicity. These studies suggest that the sequestering of RNA-binding proteins by repeat-containing foci contributes to the toxic phenotype in patients with ALS.

In patient-derived neurons, antisense oligos targeting the repeat region decreased RNA foci formation and glutamate-induced toxicity compared 
with scrambled control antisense oligos and corrected dysregulated gene expression.

Finally, a team led by Don Cleveland and John Ravits at UCSD confirmed that RNA foci-mediated neurotoxicity occurred in cells from patients with C9orf72 ALS.

Cleveland is chair of cellular and molecular medicine, a professor of medicine and of neurosciences and a member of the Ludwig Institute for Cancer Research Ltd. at UCSD. Ravits is a professor of clinical neuroscience at UCSD. The team also included researchers from the Cedars-Sinai Medical Center, the Washington University in St. Louis School of Medicine and Isis.

The foci were absent in tissues from patients with ALS who did not carry the expansions and from non-ALS controls.

In mice, intracerebroventricular injection of a mouse-specific antisense oligo was well tolerated and did not induce significant off-target changes in gene expression.

"Our next steps include screening for the best antisense molecules to take into the clinic to test for safety and efficacy. Once they are identified, we will perform the appropriate toxicology studies," said Bennett.

\section{Modeling efficacy}

Bryan Traynor, investigator and chief of the neuromuscular disease research unit at the National Institute of Aging and an author on the Neuron paper, told SciBX, "It has just been two years since the C9orf 72 gene was published. We have been able to develop the oligonucleotides so quickly that the animal models for this genetic form of the disease do not exist yet."

Mouse models for ALS are limited to superoxide dismutase 1 (Sod1)mutant animals. SOD1 mutations are the second most common cause of ALS and were first identified 10 years ago. ${ }^{6,7}$

Bennett told SciBX that “it doesn't make sense to test our antisense oligonucleotides in that model because we are dealing with a different genetic cause and different disease pathology. We need an animal model driven by this gene mutation. If one is identified, we will certainly test our molecules in it."

Philip Van Damme, an associate professor of medicine in the laboratory for neurobiology at the Catholic University Leuven, added, "We are now confronted with a potential molecular therapy, but no animal disease models to test these therapies are available yet. It is thus unclear at present how the observations in vitro including reduced RNA foci, reduced toxicity and mitigation of transcriptional changes induced by repeat expansion will relate to neurodegeneration in patients."

Frank Sieg, CSO of CuroNZ Ltd., agreed that it would be worthwhile to develop an animal model "mimicking the C9orf72 hexanucleotide repeat situation."

CuroNZ's NRP2945, a neural regeneration peptide, is in preclinical testing for severe, progressive multiple sclerosis (MS). The company said that the peptide also enhances survival in a mouse model of Sod1-mutant ALS.

Traynor noted that it will likely take another few years to design suitable animal models for C9orf72 ALS, but both he and Van Damme said that should not stop toxicity testing in animals and efficacy testing in iPS cells.

Bennett said that Isis has a broad patent estate and actively files patents to cover all preclinical work. The company has filed for patents covering the C9orf72 antisense molecules. In September, Biogen Idec Inc. received access to Isis' antisense technology for neurological indications. The companies are not disclosing the specific projects.

Martz, L. SciBX 6(43); doi:10.1038/scibx.2013.1210

Published online Nov. 7, 2013

\section{REFERENCES}

1. Sareen, D. et al. Sci. Transl. Med.; published online Oct. 23, 2013; doi:10.1126/scitranslmed.3007529

Contact: Robert H. Baloh, Cedars-Sinai Medical Center, Los Angeles, Calif. e-mail: robert.baloh@csmc.edu

2. Donnelly, C.J. et al. Neuron; published online Oct. 16, 2013; doi:10.1016/j.neuron.2013.10.015

Contact: Jeffrey D. Rothstein, The Johns Hopkins University School of Medicine, Baltimore, Md.

e-mail: jrothstein@jhmi.edu

Contact: Rita Sattler, same affiliation as above e-mail: rsattle1@jhmi.edu

3. Lagier-Tourenne, C. et al. Proc. Natl. Acad. Sci. USA; published online Oct. 28, 2013; doi:10.1073/pnas.1318835110

Contact: John Ravits, University of California, San Diego, La Jolla, Calif. e-mail: jravits@ucsd.edu

Contact: Don W. Cleveland, same affiliation as above e-mail: dcleveland@ucsd.edu

4. DeJesus-Hernandez, M. et al. Neuron 72, 245-256 (2011)

5. Renton, A.E. et al. Neuron 72, 257-268 (2011)

6. Rosen, D.R. et al. Nature 362, 59-62 (1993)

7. Deng, H.X. et al Science 261, 1047-1051 (1993)

COMPANIES AND INSTITUTIONS MENTIONED

Biogen Idec Inc. (NASDAQ:BIIB), Weston, Mass.

Catholic University Leuven, Leuven, Belgium

Cedars-Sinai Medical Center, Los Angeles, Calif.

CuroNZ Ltd., Auckland, New Zealand

Isis Pharmaceuticals Inc. (NASDAQ:ISIS), Carlsbad, Calif.

The Johns Hopkins University School of Medicine,

Baltimore, Md.

Ludwig Institute for Cancer Research Ltd., La Jolla, Calif.

Mayo Clinic, Jacksonville, Fla.

National Institute on Aging, Bethesda, Md.

University of California, Los Angeles, Calif.

University of California, San Diego, La Jolla, Calif.

University of Helsinki, Helsinki, Finland

Washington University in St. Louis School of Medicine,

St. Louis, Mo. 